\title{
Antibiotic stewardship algorithm to rationalise antibiotic use among hospitalised COVID-19 patients
}

\section{Dear Editor,}

As presentation of COVID-19 may mimic that of bacterial pneumonia, antibiotics are often prescribed. Concerns regarding overuse of antibiotics are now being raised ${ }^{1,2}$ particularly as we learn of the low rates of bacterial and fungal co-infection. ${ }^{2}$ To limit unnecessary antimicrobial exposure, we posit an algorithm for antibiotic guidance.

We developed a risk assessment algorithm using clinical biomarkers for antibiotic guidance in COVID-19 patients based on a review of the current COVID-19 treatment guidelines and medical journals in PubMed. The PubMed database was searched from 1 January 2020 to 5 May 2020 using the following search terms: "COVID-19", "SARS coronavirus", "bacterial" and "co-infection". We then embarked on a 1-day (6 May 2020) cross-sectional review of hospitalised COVID-19 patients in the National University Hospital, Singapore-a 1,200-bed tertiary teaching hospital- to determine the prevalence of antibiotic use in COVID-19 in our hospital and to examine if our algorithm was applicable. All confirmed/highly suspected COVID-19 patients admitted to designated COVID-19 isolation beds or cohort wards and who were prescribed antibiotics for pulmonary infections were surveyed. They were then followed up for 14 days postadmission/ COVID-19 positive diagnosis. This study was exempted from ethics review as it was part of an Antimicrobial Stewardship Program (ASP) quality improvement project.

We reviewed 5 COVID-19 treatment guidelines from the Infectious Diseases Society of America (last updated 5 April 2021), the National Centre for Infectious Diseases, Singapore (last revised 4 January 2021, Version 5.0), the World Health Organization (last revised 25 January 2021), Surviving Sepsis Campaign (last updated 29 January 2021) and the National Institutes of Health, US (last updated 23 April 2021). The first 2 guidelines did not address antibacterial use while the other 3 guidelines included recommendations on general management and/or principles of antimicrobial use. No algorithm on starting or withholding antibiotics was put forth.

Co-infection rates and inflammatory markers from 7 studies were tabulated. ${ }^{3-9}$ Majority of the patients in the studies were given antibiotics and had low or unreported rates of bacterial co-infections. Sicker patients generally had significantly elevated white blood cells (WBC), $\mathrm{C}$-reactive protein (CRP) and procalcitonin (PCT).

Our proposed algorithm for initiating/withholding antibiotics is shown in Fig. 1. We proposed that antibiotics should only be considered when an infiltrate is seen on the chest X-ray $(\mathrm{CXR})$ and $\mathrm{WBC} \geq 10 \times 10^{9} / \mathrm{L}$, or in severe disease requiring intensive care unit (ICU) care. We suggest withholding antibiotics when CRP is $<60 \mathrm{mg} / \mathrm{L}$ or PCT is $<0.5 \mathrm{ng} / \mathrm{mL}$. These $\mathrm{WBC}$ and PCT ranges were chosen as they are commonly associated with bacterial infections, frequently reported in the current literature and were shown to be associated with a worse outcome in SARS-CoV-2 infection. ${ }^{3-8}$ Wide ranges of CRPs have been used in the current COVID-19 literature. We chose a higher cut-off value of $\mathrm{CRP} \geq 60 \mathrm{mg} / \mathrm{L}$ as our experience suggested that a higher CRP is associated with higher severity and may be indicative of a secondary bacterial infection.

We recommend broad-spectrum antibiotics only for severely ill patients while a short duration of macrolide can be given to non-severe patients with concerning biomarkers.

On the day of the survey, the prevalence of confirmed/ highly suspected COVID-19 patients who were prescribed antibiotics was 18/117 (15.4\%). The median age was 44 and the majority were men (94\%), with foreign workers residing in dormitories forming the bulk of these patients (78\%). Sixteen patients were in the general ward while 2 were in the ICU. Common indications for starting antibiotics include communityacquired pneumonia (50\%) and fever with no CXR changes $(33 \%)$.

Based on our proposed algorithm, only 4 patients would have met the eligibility for antibiotics during the 14-day period. Among these 4 patients, 2 had non-severe disease, $\mathrm{CXR}$ infiltrates and $\mathrm{WBC} \geq 10 \times 10^{9} / \mathrm{L}$ or $\mathrm{CRP} \geq 60 \mathrm{mg} / \mathrm{L}$, and 2 had severe disease with $\mathrm{CXR}$ filtrates requiring ICU admission.

The low prevalence of antibiotic use in our confirmed/ highly suspected COVID-19 patients was likely due to our patients being a younger cohort with milder disease, and the experience our physicians developed with COVID-19 cases.

The algorithm is relevant to our population. Our general ward patients had a median WBC of $6.81-6.82 \times 10^{9} / \mathrm{L}$ 


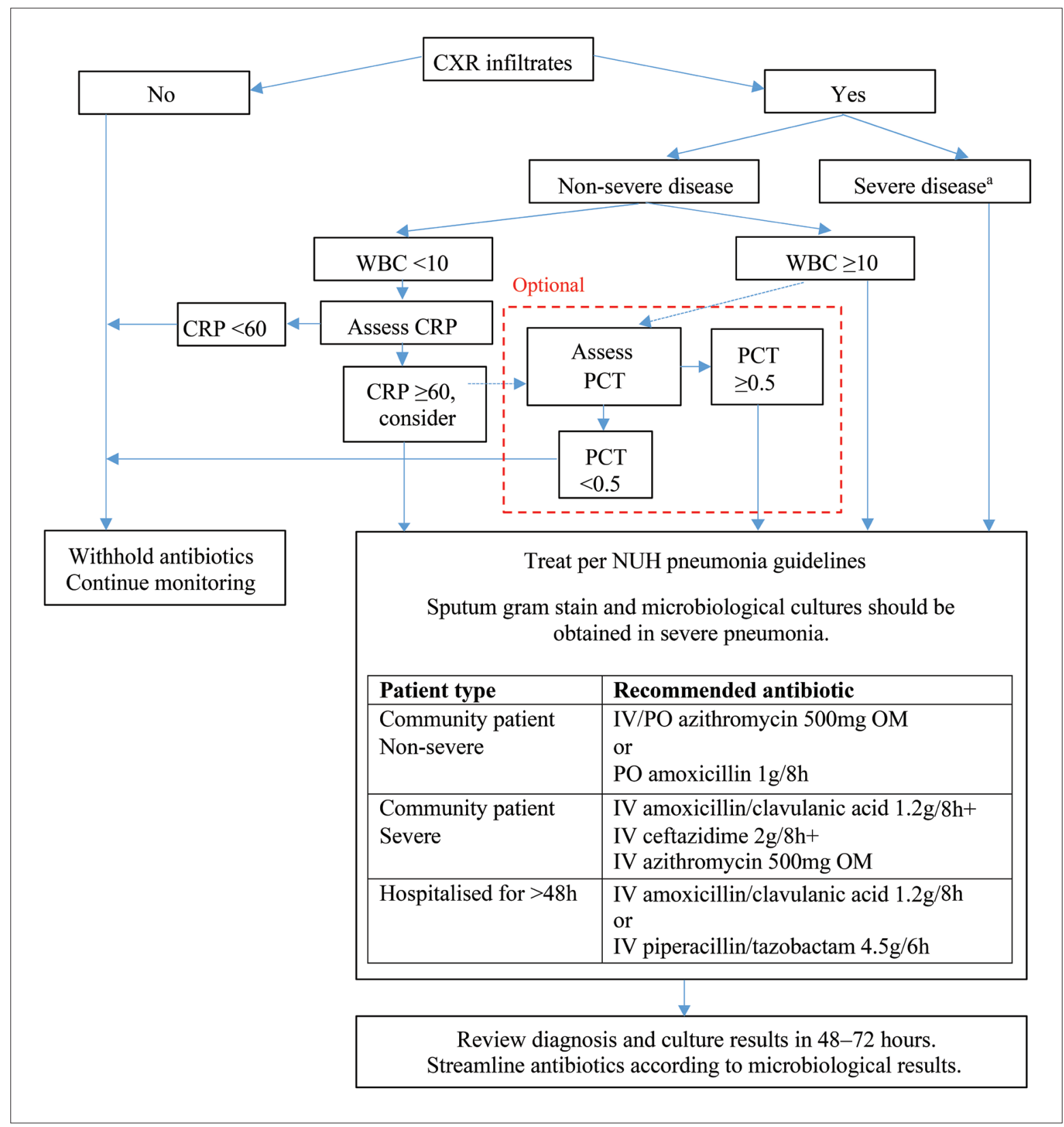

Fig. 1. Recommended algorithm for antibiotic initiation in confirmed/highly suspected COVID-19 patients.

CRP: C-reactive protein; CXR: chest X-ray; IV: intravenous; NUH: National University Hospital, Singapore; PCT: procalcitonin; WBC: white blood cells

Units: CRP in $\mathrm{mg} / \mathrm{L}, \mathrm{PCT}$ in $\mathrm{ng} / \mathrm{mL}, \mathrm{WBC}$ in $10^{9} / \mathrm{L}$

${ }^{a}$ Definition of severe COVID-19 disease

- Dyspnea: respiration rate $>30$ breaths $/ \mathrm{min}, \mathrm{PaO}_{2} / \mathrm{FiO}_{2}(\mathrm{P} / \mathrm{F})$ ratio $<300$, lung infiltrates $>50 \%$ of lung fields within $24-48 \mathrm{~h}$

- Admission to ICU

- Currently on mechanical invasive and/or non-invasive ventilation or IV vasoactive medications to maintain mean arterial pressure $>65 \mathrm{mmHg}$

- Myocarditis/myocardial dysfunction secondary to SARS-CoV-2

and median CRP of $22-41 \mathrm{mg} / \mathrm{L}$ while the ICU patients had a higher median WBC of $7.39-9.54 \times 10^{9} / \mathrm{L}$ and median CRP of $133-235 \mathrm{mg} / \mathrm{L}$. PCT test was not done routinely. We recommend it as an adjunct to WBC to determine bacterial co-infection.
Based on our antibiotic use algorithm, 14 out of 18 $(77.8 \%)$ patients may not have required an antibiotic prescription. The current profile of our COVID-19 patients is that the majority are well (non-severe). Febrile patients from the general ward had negative 
cultures when these were requested. We believe that the antibiotic use in these patients can be reduced further, and may particularly benefit COVID-19 patients who do go on to develop a more complicated and often prolonged course of the disease.

To our knowledge, this is the first study in Singapore that proposes an antibiotic use algorithm in the treatment of COVID-19 patients. Our strategy is to limit patients' exposure to antibiotics in the early course of SARS-CoV-2 infection, thus reducing their risk of acquiring a multidrug-resistant organism. If they subsequently deteriorate and require admission to the ICU, viable options may still include broad-spectrum beta-lactams such as amoxicillin/clavulanic acid or piperacillin/tazobactam, thus circumventing immediate escalation to carbapenems. Antibiotics should generally be withheld if suspicion for COVID-19 is high, or stopped in patients whose COVID-19 swabs return positive with no clear evidence of bacterial superinfection. Based on our study and others, co-infection is less likely until much later in the hospital stay. ${ }^{3,9}$

COVID-19 presents antibiotic stewardship with challenges, and the onus is on every ASP team to seize the opportunities to optimise antibiotics use in the face of a constant evolving body of evidence. The existing COVID-19 treatment guidelines are diverse and may not always address antimicrobial stewardship issues. Stevens et al. identified multiple potential areas where ASPs can support emergency response efforts. ${ }^{10}$ Research efforts such as evaluating the use of empiric antimicrobial agents in patients with COVID-19, assessing collateral damage from use of antibiotics in COVID-19 patients, and identifying risk factors of patients with subsequent bacterial or fungi infection can be undertaken.

Our inpatient population at the time of the survey were generally patients with mild COVID-19 cases. The 1-day survey may not be representative of all COVID-19 patients. Safety outcomes such as mortality and length of stay were not assessed. Larger-scale studies are needed to assess the validity of the algorithm and its use in other settings.

The prevalence of prescribing antibiotics in our hospital for confirmed/highly suspected COVID-19 patients was a relatively low $15.4 \%, 3$ months into Singapore's COVID-19 outbreak since the first case was confirmed on 23 January 2020. Using our proposed algorithm, we can potentially reduce the rate of prescribing antibiotics further. We suggest using inflammatory markers such as WBC, CRP and PCT, in addition to CXR, to aid early risk assessment to guide the initiation of antibiotics. Undue antibiotic prescribing in COVID-19 may add to the ubiquitous challenge of increasing antimicrobial resistance. Antibiotic stewardship principles should continue to be applied and promoted even in these challenging times.

\section{REFERENCES}

1. Huttner BD, Catho G, Pano-Pardo JR, et al. COVID-19: don't neglect antimicrobial stewardship principles! Clin Microbiol Infect 2020;26:808-10.

2. Rawson TM, Moore LSP, Zhu N, et al. Bacterial and fungal co-infection in individuals with coronavirus: A rapid review to support COVID-19 antimicrobial prescribing. Clin Infect Dis 2020;71:2459-68.

3. Zhou $\mathrm{F}, \mathrm{Yu} \mathrm{T}, \mathrm{Du} \mathrm{R}$, et al. Clinical course and risk factors for mortality of adult inpatients with COVID-19 in Wuhan, China: a retrospective cohort study. Lancet 2020;6736:1-9.

4. Wang D, Hu B, Hu C. Clinical characteristics of 138 hospitalized patients with 2019 novel coronavirus-infected pneumonia in Wuhan, China. JAMA 2020;323:1061-9.

5. Chen $\mathrm{T}, \mathrm{Wu} \mathrm{D}$, Chen $\mathrm{H}$, et al. Clinical characteristics of 113 deceased patients with coronavirus disease 2019: retrospective study. BMJ 2020;368:m1091.

6. Ruan Q, Yang K, Wang W, et al. Clinical predictors of mortality due to COVID-19 based on an analysis of data of 150 patients from Wuhan, China. Intensive Care Med 2020;46:846-8.

7. Guan $\mathrm{W}, \mathrm{Ni} \mathrm{Z}, \mathrm{Hu}$ Y, et al. Clinical characteristics of coronavirus disease 2019 in China. N Engl J Med 2020;382:1708-20.

8. Goyal P, Choi JJ, Pinheiro LC, et al. Clinical characteristics of COVID-19 in New York City. N Engl J Med 2020;382:2372-4.

9. Bhatraju PK, Ghassemieh BJ, Nichols M, et al. COVID-19 in critically ill Patients in the Seattle region - Case Series. N Engl J Med 2020;382:2012-22.

10. Stevens MP, Patek PK, Nori P. Involving antimicrobial stewardship programs in COVID-19 response efforts: All hands-on deck. Infect Control Hosp Epidemiol 2020;41:744-5.

Hui Hiong Chen, ${ }^{1}$ MPharm, Gladys WT Chung, ${ }^{1}$ MClinPharm, Jia En $\underline{\mathrm{Wu}},{ }^{1}{ }_{M C \text { ClinPharm, }}$ Geraldine TT $\underline{\text { Foo, }},{ }_{\text {BSc (Pharm) (Hons), }}$ Jyoti $\underline{\text { Somani }},{ }^{2}{ }^{2}$

\footnotetext{
${ }^{1}$ Pharmacy, National University Hospital, Singapore

${ }^{2}$ Division of Infectious Diseases, Department of Medicine, National University Hospital, Singapore
}

Correspondence: Dr Jyoti Somani, Division of Infectious Diseases, Department of Medicine, National University Hospital, 5 Lower Kent Ridge Road, Singapore 119074.

Email: somani_jyoti@nuhs.edu.sg 